\title{
Importancia del branding para consolidar el posicionamiento de una marca corporativa
}

\section{Importancia del branding para consolidar el posicionamiento de una marca corporativa}

\author{
Ronald Leodan Maza Maza*1, Benito Danilo Guaman Guaman ${ }^{1}$, Alexandra Margarita Benítez Chávez ${ }^{1}$, Gustavo \\ Solis Mairongo ${ }^{1}$ \\ ${ }^{1}$ Universidad Laica Eloy Alfaro de Manabí, Ecuador \\ *mazaronaldm01p.basicos@gmail.com
}

DOI: https://doi.org/10.26871/killkanasocial.v4i2.459

\begin{abstract}
Resumen
Este artículo realza la importancia del branding para consolidar el posicionamiento de una marca corporativa. El presente estudio fue aplicado a los usuarios del Consultorio Fisioterapéutico KAMB y a una muestra de habitantes cercanos a la entidad, con la finalidad de conocer los criterios de percepción que ellos tienen sobre la marca. La problemática consiste en que el consultorio tiene una identidad visual que se creó de manera empírica y bajo la presión de tener un nombre comercial, además hay un escaso profesionalismo en el manejo de la marca, puesto que su logotipo y estructura publicitaria no concibe la esencia de la empresa. Por ello, el objetivo general es analizar la importancia de la renovación de una marca corporativa para el Consultorio Fisioterapéutico KAMB de la ciudad de Manta, mediante el uso de recursos estratégicos de branding que permitirá proyectar su posicionamiento en el mercado. Por esta razón se realizó una investigación de tipo cuantitativa, aplicando la técnica de la encuesta que estuvo divida en dos secciones; una para los pacientes y otra para los ciudadanos. Según los resultados arrojados por la investigación, existe un mínimo posicionamiento donde la gran mayoría de personas encuestadas, dicen no reconocer la marca, a más de ello, los usuarios y la población consideran contundentemente que esta debe pasar por un proceso de renovación, para que se diferencie de la competencia y al mismo tiempo se convierta en algo fresco, innovador, expresivo e interactivo, que tenga presencia, reconocimiento y estética visual, puesto que la actual no representa estas características. Es necesario que se plantee una estrategia de branding para proyectar, fortalecer y darle valor a la marca.
\end{abstract}

Palabras clave: branding, marca, posicionamiento, renovación, identidad, consultorio fisioterapéutico KAMB.

\begin{abstract}
This article highlights the importance of branding to consolidate the positioning of a corporate brand. The present study was applied to the users of the Physiotherapy Clinic KAMB and to a sample of inhabitants close to the entity, with the purpose of knowing the criteria of perception that they have on the brand. The problem is that the office has a visual identity that was created empirically and under the pressure of having a trade name, in addition there is little professionalism in the management of the brand, since its logo and advertising structure does not conceive the essence of the company. Therefore, the general objective is to analyze the importance of the renewal of a corporate brand for the Physiotherapy Clinic KAMB in the city of Manta, through the use of strategic branding resources that will project its positioning in the market. For this reason a quantitative research was carried out, applying the technique of the survey that was divided into two sections, one for patients and another for citizens. According to the results of the research, there is a minimum positioning where the vast majority of people surveyed say they do not recognize the brand, moreover, users and the population strongly believe that this must go through a process of renewal, to differentiate it from the competition and at the same time become something fresh, innovative, expressive and interactive, which has presence, recognition and visual aesthetics, since the current does not represent these characteristics. It is necessary to consider a branding strategy to project, strengthen and give value to the brand.
\end{abstract}

Keywords: branding, brand, positioning, renewal, identity, physiotherapy clinic KAMB.

\section{Introducción}

El tema "La importancia del branding para consolidar el posicionamiento de una marca corporativa" tiene como objetivo principal, analizar la importancia de la renovación de una marca corporativa para el Consultorio Fisioterapéutico KAMB de la ciudad de Manta, mediante el uso 
de recursos estratégicos de branding para proyectar su posicionamiento en el mercado. Por ello, es importante en primer lugar, determinar el posicionamiento de la marca en la mente de la ciudadanía e identificar la cantidad de los habitantes que la reconocen, sobre todo considerar la utilización de la estrategia de Branding para potencializar la identidad e imagen corporativa.

El branding es un proceso que construye y maneja estratégicamente las marcas, es decir cómo estas se quieren proyectar al público.

Es fundamental aclarar que una marca no es un logotipo, tampoco es una empresa, de hecho, solo son unos indicadores de identidad; pero se convierten en marca cuando estos cumplen con ciertas características como: transmitir atributos y valores descubiertos por los insight (verdad interna), que forman y consolidan la personalidad de una marca; donde por medio del uso de estrategias de marketing, comunicación, publicidad y branding, permiten crear las destrezas necesarias para proyectarla en la mente de los consumidores.

La marca se vuelve tangible por medio de su logotipo, al ser un conjunto de elementos como; el símbolo, icono, y slogan, es decir que se pueden ver, tocar, oler y escuchar, es un rito que conecta con el cliente. Sin embargo, la renovación es un proceso donde se busca que un objeto se reanude o se reestablezca encontrando primero su esencia y en base a eso generar las estrategias de branding idóneas para trasmitirla a los demás.

El Consultorio Fisioterapéutico KAMB de la ciudad de Manta tiene 10 años brindando servicios de fisioterapia y durante los últimos cinco años, una sola vez ha sufrido leves cambios en la estructura publicitaria que nació de manera empírica a base de la necesidad de tener rápidamente una identidad, lo cual ha generado que la proyección de su marca corporativa no corresponda a su esencia empresarial. Por ello, es trascendental el uso de estrategias de branding para lograr el fortalecimiento y posicionamiento en el mercado.

El presente estudio fue realizado de manera cuantitativa mediante la aplicación de la técnica de la encuesta, a los usuarios y habitantes cercanos al objeto de estudio. De acuerdo a los datos reflejados en la investigación, es necesario que KAMB fortalezca su imagen e identidad y la renovación es una oportunidad para el consultorio en estos momentos; donde el servicio profesional es muy bueno solo que le hace falta establecer una estrategia de branding para que la marca comunique y se proyecte su valor hacia el futuro, de modo que ocupe un lugar en la mente todos los ciudadanos.

Este artículo tiene como fundamento aportar con nuevos estudios direccionados al área de las ciencias sociales, y al campo de las actividades como el marketing y branding, para dar a conocer la importancia de la proyección del valor de una marca, donde el consumidor busca nuevas y novedosas alternativas que logren conectar con sus emociones.

\section{Marco teórico}

\subsection{El branding}

\subsection{Dato histórico del branding}

Según (Velilla, 2010), el branding es un término de origen Anglosajón que cuya aplicación ha ido cambiando a lo largo de la historia global. La palabra Brand es originaria del idioma escandinavo (Brandr) que quería decir quemar y antiguamente esta era una técnica que se utilizaba para colocar una especie de marca utilizando el fuego en objetos y ganado, como una forma de preservar su identidad y no sea fácil de falsificar. Esa misma estrategia se mantuvo en plasmar un sello de identidad en las vasijas (etruscas), griegas o romanas o en la porcelana fina francesa del siglo XVII.

Para (Santamaria Luiggi, 2013), luego del crecimiento del mercantilismo y posterior a la Segunda Guerra Mundial nace la llamada revolución industrial donde se producía bienes a grandes escalas para satisfacer la alta demanda de consumidores y era necesario que los productos tuvieran su propia marca para que pudieran ser diferenciados unos de otros.

La revolución industrial marca un antes y un después en la historia de las marcas. Sin embargo, es cuando llega el siglo XX entre 1870-1920 más específicamente, las empresas comenzaron a implementar otro tipo tecnologías para producir a gran escala y en serie. Es aquí que el Branding comienza a tener gran acogida y representación porque aparecen las grandes marcas que muchas de ellas aún siguen en el mercado actual como "Coca Cola, Kodak, American Express, Kelloggs, Colgate" por citar algunos ejemplos (Velilla, 2010, p. 26)

Empresas que ya comenzaron a expandir su marca no solo en sus productos sino también en los vehículos de reparto, documentos, etc. Estas empresas tenían la necesidad de mostrar desde otra perspectiva sus productos ya sea de forma llamativa o promocionando sus marcas (Delgado Gentili Luis Armando, 2012)

(Santamaria Luiggi, 2013), plantea que en los años 90 el Branding se convierte en una herramienta primordial del Marketing puesto que las marcas vendían a los consumidores y con la llegada de la nueva tecnología y con ella el internet todo se transformó. El branding tuvo que renovarse a la realidad actual y por ende debió cambiar la forma de hacer marketing puesto que las sociedades ya tenían otras necesidades que satisfacer. Entonces el branding tuvo que ajustarse a conocer un poco más al consumidor, brindarle la importancia necesaria para saber lo que le gusta o le que le disgusta y de esta forma delimitar que el consumidor se encuentra en constante evolución.

\subsection{Definición de Branding}

Según lo plantea Ilgo (2019), branding es una herramienta del marketing que funciona como una planificación estratégica que comprende de forma general la creación, manejo, posicionamiento y funcionamiento de una marca. 
"Las marcas no son para ti si no para los consumidores" (p.46). Las marcas viven en la mente del público o consumidor, es por ello que existe una gran batalla por parte de las empresas para lograr llegar primero a la mente del consumidor mediante la proyección de una marca, debido a que todo el tiempo está siendo rodeado por ellas y como tal existe un gran apogeo de percepciones con relación a ellas.

En cambio, (Hoyos Ballesteros, 2016), manifiesta que "el branding es un proceso de acción para colocarle un nombre (naming) a un producto o servicio". Que se encarga de construir marcas imponentes, que sean conocidas y que sobresalgan en el mercado globalizado, que sean asociadas con aspectos positivos que tengan valores y sean deseadas por una gran mayoría de usuarios. (p.1)

Por lo tanto, se puede concluir que el branding es una herramienta estratégica interactiva dentro del marketing que ha tomado una fuerza muy grande en los últimos años, puesto que se le ha dado la importancia necesaria. Se utiliza para construir y gestionar de forma integral una marca para adecuarla al contexto donde esta se desarrolla. De forma que permita capturar la esencia, crear una personalidad, fortalecer una identidad gráfica y trabajar con ello para crear una marca más valiosa, poderosa, diferente y que conecte con las emociones de los consumidores.

\subsection{Tipos de Branding}

Existen diversos tipos de branding conforme los mencionan los siguientes autores.

\subsubsection{Branding Personal}

Matthews citado por (Velilla, 2010) dice que una marca es lo que la gente percibe de tí. En la actualidad las sociedades han evolucionado en muchos sentidos y en este caso como seres humanos se han permitido cada uno en tener sus propias características muchas de ellas similares y otras que son totalmente individuales. Que marcan una diferenciación y esto ha permitido que los seres humanos quieran tener su propio sello característico denominado marca personal.

Significaría trabajar mucho con el individuo en cuanto a la búsqueda de su propio "YO" interior, sus fortalezas y encontrar ese valor que se convertirá en esa diferencia que lo hará distinto a los demás. Una marca personal tiene que tomarse su tiempo para ser desarrollada debido a que se basa en la percepción que tiene el uno del otro. Se debe sacar lo mejor de sí mismo, porque el valor de cada ser humano está dado por sus habilidades profesionales.

Sin embrago para (Perez Ortega Andres, 2008), La marca personal se encuentra en entre los requisitos más importantes de una persona debe tener. Esta debe ser una herramienta con la que pueda transmitir sus conocimientos, seguridad, profesionalismo, innovación y buenas relaciones sociales para que de esta manera pueda liderar como tal en la mente de las demás personas.

\subsubsection{Branding Sustentable}

Para (Hernandez White Raquel, 2012), el branding sustentable enfoca a una marca para que conozca el daño o beneficio está generando indirectamente en su contexto social, para ello debe aportar a buscar un estático punto de equilibrio y amigable en tres aspectos fundamentales, el medio ambiente, la sociedad y la economía, con una profunda raíz hacia la conciencia colectiva donde cada día establece normas de comportamiento aplicacando valores éticos, morales y o económicos a largo plazo. El branding actual debe conocer el progreso y las transformaciones de los mercados, consumo, tendencias, comportamiento social y ambiental para generar estrategias afines para conseguir un desarrollo sostenible y el posicionamiento en ese sentido.

\subsubsection{Branding Estratégico}

(Alejandro Razak, 2019), menciona que este tipo de branding se enfoca en dar valor a los productos por medio de la marca que sea perdurable en la línea de tiempo y que ofrezca beneficios colaterales de la funcionalidad del producto.

Mientras que (Soza Angie, 2019), expresa que el branding estratégico es la construcción, elaboración y ejecución de los activos de una organización. Busca crear valor a la marca desde la perspectiva del consumidor donde se le brinda un bien o servicio en base a sus beneficios que además se incluyen el comportamiento personal que son los que generan la reputación de la organización, hacia los públicos externos como los proveedores que manejan tratos justos y que confían en la marca, finalmente a los grupos de interés que representan a la marca.

A diferencia (Capriotti, 2010), el branding estratégico también se denomina corporativo, es la creación de una imagen corporativa que se desarrolla en base a la generación de valor hacia la empresa. Comenzando por delimitar la razón de existencia de la compañía, la filosofía, valores y cultura organizacional además de sus productos o servicios que vayan de la mano con los puntos antes mencionados. Que se sustenta en lo estratégico mediante la proyección de tres ejes fundamentales que son la identidad, imagen y comunicación corporativa.

\subsubsection{Branding Emocional}

Para (Soza Angie, 2019), este tipo de branding conecta al público con la marca mediante las emociones siguiendo una serie de tácticas para que se genere expectativas emocionales porque el consumidor en la actualidad no busca solo cubrir sus aprietos sino también es necesario que exista una experiencia de compra se sobreponga a sus expectativas.

Concuerda con (Caceres Gutierrez Amparo, 2012), que señala al branding emocional como una técnica de comunicación que ayuda a plantear elementos que conciben a llevar el proceso de reconocimiento de una marca para que esta genere un impacto y percepciones en los usuarios. 
Así mismo para (Valiente Alber, 2016), el branding emocional debe manejar los insight (visión interna) del usuario, un término que proviene de la lengua inglesa relacionado con la psicología. Es funcional en los siguientes aspectos penetración, percepción, o motivación que permite conocer de forma general el pensamiento, intuición, deseos y conducta de los consumidores.

Complementando el tema de los insights es algo que busque una diferencia mucho más allá de mostrar solo los beneficios funcionales o emocionales visibles de un servicio. Si no, más bien, estos deben encontrar esa relación más íntima que conecte al usuario con el producto.

\subsection{Elementos del branding}

La comunicación es un hecho real dentro de la publicidad, un mecanismo del cual toma referencia el branding para desarrollar estrategias creativas situadas en generar mensajes de forma apropiada para introducirlos en la mente de los consumidores, el hacer una marca construye en primera instancia en conocer fortalezas y debilidades internas de la organización para así poder descubrir la esencia de existencia de la empresa a fin de poder transmitir el significado de una identidad al target. (Valenzuela Tamayo Alejandro, 2013)

Según Meldini (2015), existen cinco elementos principales para construir una marca de una empresa u objeto, los cuales son:

\subsubsection{Naming}

El primer paso consiste en crear un nombre o una denominación para posteriormente construir una identidad de marca que permita que la empresa, producto o servicio se compacte y se diferencien del resto. Si es en el contexto institucional o empresarial el naming colabora a construir un nombre que genere una sensación al momento de escucharlo por los demás; ya sea por su fonética, palabras, formas o su originalidad. (Meldini Adriana, 2015)

Se podría recurrir a la búsqueda de ciertas palabras, conocidas que se generan en conversaciones cotidianas, que se consideran bellas en su forma literaria que al ser expresadas responden a un mecanismo apropiado de acuerdo al significado de nuestro lenguaje recuerdan o simulan alguna experiencia al ser nombradas son algunas alternativas que presenta el autor al momento de crear un nombre. (Grau, 2011)

Siguiendo con el planteamiento que los autores describen anteriormente, la existencia del naming sienta un precedente muy relevante en la creación de un nombre comercial, que para su elaboración pasa por procesos técnicos que pertenecen al branding, de modo que cumpla con características como el aportar valor a la marca, ser escuchada, hablada, vista de forma positiva para que genere recordación en los públicos.

\subsubsection{Identidad Corporativa}

Según (Duarte y Contreras, 2014), la Identidad Corporativa es un conjunto de características internas que generan la personalidad de la empresa; se mantienen en vigencia y la diferencian en el entorno donde se desarrolla. La identidad se comunica a través de su imagen mediante elementos como: conducta, imagen y comunicación que funcionan como objetos de comunicación externa, hacia lo públicos objetivos. Mientras la esencia de la organización es aquel concepto los acompaña y juntos forman una sola identidad corporativa.

A sí mismo, (Zapana y Salvador, 2018) menciona que la identidad corporativa se asocia a la gestión interna que la empresa realiza en cuanto a su filosofía, decisiones, estrategias, reglamentos y a sus acciones en concreto que realiza frente a los públicos.

Dentro de la identidad se presentan dos componentes que forjan la Identidad Corporativa

- La identidad Visual Según lo define (Olvera y Zambrano, 2019), la identidad visual es el proceso de percepción visual que un ser humano tiene sobre un símbolo existente; que se genera mediante la abstracción mental de su diseño y la extensión de cada uno de los componentes a simple vista y que diferencian a la organización de otra. La identidad visual tiene que caracterizarse por ser muy definida, coherente, única y exclusiva.

- $\quad$ La identidad Cultural (Lueza, 2018), señala que con un enfoque más general la identidad cultural abarca rasgos internos como las creencias y formas de interrelación con los demás. En términos más generales está dirigida por la personalidad de la organización marcada por valores, misión y visión que define la existencia de la empresa. Puesto que además se ve reflejada en cada uno de sus colaboradores a si mismo ayuda a llevar un orden en los aspectos internos y buscar una adaptación en el medio donde se desarrolla la organización.

\subsubsection{Posicionamiento}

(Meldini Adriana, 2015), el posicionamiento es un término que indica en qué lugar se encuentra la marca de un producto o servicio, en la mente de los usuarios. Por ello se importante encontrar una cualidad o atributo de la cual hará un prototipo para que un sello se apropie de un mercado de mentes humanas.

Para desarrollar un posicionamiento y por ende la fidelización es necesario conocer el target o público objetivo y esto se logra con una segmentación de mercado que le permite al estratega estudiar al consumidor y delimitar al público al que va dirigida la marca y sus derivados. (Alicia Anabel Aucapiña Alvarado, 2017)

A sí mismo, el posicionamiento debe ir en base a un valor o cualidad a largo plazo que se le atribuye a la marca y que no tiene final puesto que, es por ello que la recordaran siempre. 


\subsubsection{Lealtad hacia la marca}

Según Capriotti citado en (Meldini Adriana, 2015), una marca es una promesa que debe cumplir a satisfacción con lo que dice ser". Porque de ello depende las futuras asociaciones o calificativos que se le adhieran a la misma debido a una buena o mala experiencia que hayan vivido uno o varios usuarios frente al sello que se convierte en una garantía del producto o servicio. (p.26)

Todo funciona como una gran red que se conecta de forma especial entre usuarios y marcas cuando ha existido cumplimiento de la promesa. Este ciclo sucede en la mente de los usuarios que proyecta cualidades positivas o negativas debido a las experiencias de compra que ha tenido. Lo cual trae la lealtad del cliente porque el busca un sello de garantía que le genere confianza hacia la marca para después convertirla en la de su preferencia.

\subsubsection{Arquitectura de la marca}

Es aquella estrategia que permite al branding definir con claridad y coherencia cinco elementos que muestran la esencia de la marca. Estos son: identidad, misión, visión, valores y cultura corporativa. Que sería aconsejable poner en práctica en una empresa tanto de forma interna con sus empleados o de forma externa con sus usuarios, proveedores y medios de comunicación. (Meldini Adriana, 2015)

Para (Meldini Adriana, 2015), establecer una arquitectura de marca implica plasmar una estructura que funcione como guía en el futuro que responda a ciertos cuestionamientos como la creación de unos nuevos productos, nuevos mercados, enfoque de la comunicación y publicidad y además sea un respaldo de las decisiones que le empresa tome en un futuro.

Es decir, la arquitectura de la marca funciona como un mecanismo que responder a su filosofía corporativa y a la cultura corporativa de la empresa para mostrar su esencia consolidada y que forme un sólido respaldo de las futuras decisiones de la empresa con respecto a la forma de actuar y como se muestra en cuanto al comportamiento tanto interno como externo y con los medios de comunicación.

\subsection{Marca}

\subsubsection{Definición de Marca}

(Medina-Aguerrebere, 2014) explica lo siguiente "[... ] La marca transmite todos aquellos elementos intangibles que la diferencian de la competencia [...]" (p.5), donde pone en evidencia los detalles visuales que se consolida como una identidad diferenciadora ya sea de un producto, servicio o de la misma empresa que los oferta.

De tal manera que la marca termina protegiendo al producto y siendo una garantía de los estándares de calidad para el usuario que interrelaciona situaciones mediante las experiencias emocionales. Tomando en cuenta que una marca no es sinónimo de un producto; sino que se convierte en la identidad y a la vez en la esencia del mismo.

Las marcas son el motor que coadyuva al surgimiento de las empresas como sellos que representan al producto y se busca que exista una identificación con los usuarios de esta manera lo interpreta. (Olins, 2015)

(Velilla, 2010), la marca es una huella visual, emocional, cultural y una promesa que permanece a través del tiempo en la mente de los consumidores que refleja la esencia, personalidad, experiencias y o beneficios que finalmente ofrece una garantía a los usuarios. La marca es la identidad, el alma de las organizaciones y también de los productos y servicios que ofertan.

\subsubsection{Las marcas y sus signos}

Una marca no es un logo, una identidad ni un producto; generalmente concebimos erróneamente los conceptos, pero estos términos trabajan conjuntamente para conseguir un fin, donde cada uno de ellos tiene un significado diferente. Las marcas se han convertido en parte de la cotidianidad de los seres humanos convirtiéndose entonces en un rasgo de nuestra cultura; incluso actualmente se busca que los consumidores se vean representados en ellas y que causen emociones ((Velilla, 2010).

En cambio (Flores Calderon Blanca, 2015), recalca que las marcas no son un producto, son un estilo de vida que genera valores, sentimientos, una experiencia que trasmite, y que se posiciona en la mente de los consumidores como tal inclusive se daría por hecho que la marca pudo insertarse dentro de una cultura previamente establecida y estudiada. "Las marcas son un fenómeno cultural "para eso hay que con conocer al usuario y la marca debe adaptarse a la nueva realidad de las sociedades. (Olins, 2015)

Las marcas están inmersas bajo su propia identidad dentro de nuestra cultura y se han visto en la necesidad de renovarse, cultivarse y trascender para convertirse en las marcas poderosas y competitivas. Donde se reconoce que ya no son, solo un producto o un logo; si no que es necesario que trasmita su esencia, que tenga presencia en el mundo y genere nuevas emociones para crear una relación mediante la experiencia esta sea más cercana a los consumidores, y estos las lleven a formar parte de sus vidas.

Según (Velilla, 2010), señala que existen cuatro signos relevantes a tomar en cuenta para conformar una marca.

\section{- Signo Verbal}

(Velilla, 2010), el desarrollo de una marca se individualiza en un primer aspecto para generar una personalidad en la empresa, en la denominación del nombre, un aspecto verbal que guarda una relación directa y estrecha con los consumidores, este opera como un índice pronunciable que de armonía al escucharse y que conlleva un significado de experiencia agradable. Para que un signo verbal sea algo diferente, universal y único debe tener ciertos atributos como los semánticos, que ofrecen un conjunto de significados que se relacionan mediante las asociaciones que se le atribuyen a una marca como palabra propiamente creada y percibida por el consumidor. También están los atributos fonéticos que se refiere a toda forma de sonidos silábicos y rítmicos que son evocados bajo la pronunciación de la marca. De igual manera los morfológicos que permiten realizar una 
conformación y estructura interna de los elementos que conforman la palabra o nombre con cierta ubicación que al ser percibidos se note agradable.

Finalmente, los atributos de marketing que cumple varias funciones estratégicas para que la marca sea única tenga personalidad, sea identificada, recordable, moldeable y aplicable, y principalmente que todo vaya acorde al concepto que se quiere posicionar en el mercado.

\section{- $\quad$ El logotipo}

Está conformado por todos aquellos signos visuales que manifiestan mediante su composición (colores, tamaño, tipografía) el nombre de una marca. (Velilla, 2010),

El logotipo es una representación de la marca visible porque se puede leer y perceptible a través del sonido puesto que emite caracteres fonéticos. Es una unidad que comunica el concepto del existir de una empresa. (Ortegón Costázar, 2014)

Cuando se habla de logotipo es necesario poner en consideración los siguientes conceptos:

Gama Cromática: Según (Evangelista y Baca, 2018), el color es una sensación captada a escala general por el ojo humano que percibe reflejos hacia su cerebro. Una gama de colores es un conjunto de variaciones de colores cuyas composiciones se clasifican en base a la luz que reflejan colores primarios (amarillo, azul y rojo), secundarios (violeta, naranja y verde), y terciarios son aquellos que se obtiene de la mezcla entre un primario y secundario. La gama cromática está compuesta por tonos fríos que abarcan desde la tonalidad azul y los cálidos que toma en consideración las tonalidades rojo y sus diferentes variaciones.

Es por ello que el color es un elemento importante dentro del diseño, porque permite elevar un significado cuando se habla de la creación de marcas y la gama de colores presenta una propuesta extensa de luces, contrastes y tonalidades de las cuales se puede combinar para conseguir un color en específico.

Tipografía: "La tipografía es un sistema de objetos sensibles especialmente potente" (Lupton y Phillips, 2016), abstraer una forma que tienen las letras es una actividad que se interioriza mediante los sistemas de lectura donde se presentan palabras con distintas asimetrías que guardan lineamientos rectos, oblicuos, curvos que en el diseño se le dará un sentido y que sea percibida en base a relaciones que tiene con el producto o la marca. (p.100)

Símbolo: Es un icono asociado a la marca según lo plantea (Hoyos Ballesteros, 2016), esto puede variar de acuerdo a la naturaleza de la empresa, esta puede ser una mascota, un personaje que tenga alguna relevancia o forme parte de la creación de la marca. (p.30)

De modo que (Ortegón Costázar, 2014), visualiza al símbolo como un conjunto de caracteres icónicos perceptibles representados u asociados a la marca las cuales están concebidas por medio de imágenes aceptadas dentro de una sociedad y que tienen su propia identificación.

- $\quad$ El producto y su imagen
Es un elemento de reconocimiento y diferenciación frente a la competencia. Según (Publicaciones Vertice S. L., 2008), un cliente en la actualidad es muy exigente porque desea alcanzar mejores expectativas y con ello busca la calidad que es un factor determinante para que un cliente considere la obtención de los servicios o productos que una empresa oferta. El servicio tiene que ser ese valor añadido que ayude a sustentar la presencia de la marca en el mercado.

Slogan: Es un sintagma de forma textual que va añadido a la marca y que genera un concepto más armónico sobre a la marca.

Para (Del-Rio, Cardona-Arbelaez, y SimancasTrujillo, 2017), el lema comercial o slogan va de forma cercana la marca, es su distintivo que la complementa y consolida su razón de ser. Un mecanismo que se efectúa mediante una frase o conjunto de palabras que permiten reforzar la recordación en la mente los públicos hacia los cuales está orientada la marca su producto o su servicio.

- Valor de la marca

Para (ILGO, 2019), existen 5 puntos principales que se deben tomar en consideración cuando se quiere que la marca cree valores.

La claridad porque una marca debe tener coherencia, ser concisa y consiente de lo que está representando. En otras palabras, tiene que tener muy definida su personalidad en base a términos de calidad.

Tiene que ser única que se base en tener exclusividad y algo nuevo, novedoso y fresco que mostrar al público.

Elementos que se asocien a la marca, como primer paso hay que definir el público objetivo, en base a las necesidades que satisface la marca en un mercado concreto de consumidores. Porque pasaría a formar parte de su vida.

Generar experiencias para lograrlo una marca debe ser cercana a los públicos y saber crear momentos agradables para el consumidor y su entorno.

Una marca que tiene participación en el mercado debe hablar alto, fuerte y claro. Producir confianza y su promesa básica debe apuntar hacia ese objetivo. Un usuario se siente a gusto cuando utiliza marcas conocidas puesto que genera un sustento del porqué realizo la compra.

\subsubsection{Manual de Identidad Corporativa}

Es un detalle en cual se proyecta un conjunto de directrices elaboradas en base a un lineamiento o un criterio gráfico, que sirve para orientar a toda una empresa hacia el reconocimiento de una identidad e imagen corporativa, a cargo de todo el manejo de la marca (Cubur Luis y Fernando, 2015).

Es un documento que lleva registrado un reglamento en el cual consta la constitución, elaboración y manejo de un logotipo que sustenta la existencia de una marca y también de todos significados de los elementos que se acoplan al mismo más bien dicho a la como se debe aplicar la identidad de una liana gráfica en diferentes soportes. (Hernandez Daniel, 2017) 


\subsubsection{Imagotipo}

En una composición visual de carácter imagen acompañado por un texto. (Hernandez Daniel, 2017)

Para (Cubur Luis y Fernando, 2015), el imagotipo no permite que la imagen se separe del nombre o logotipo, ambos conforman una sola composición.

\subsubsection{Isotipo}

Es un icono representativo, que mediante su percepción se puede reconocer a la empresa, no existe la necesidad de que sea acompañado de alguna franja textual, debido a que el Isotipo es un signo que no es hablado, pero por si solo representa simbólicamente a la organización. (Hernandez Daniel, 2017)

\subsection{Proceso para construir una marca}

Para (ILGO, 2019), la marca debe conectar con el público, generar reacciones, perdurar en el tiempo y ser reconocida "poderosa" en términos generales.

Para construir una maca según (ILGO, 2019), hay que seguir los siguientes pasos:

Paso 1: Estudiar el mercado donde se desarrolla la marca, el escenario externo para conocer y definir el público objetivo, saber el comportamiento (consumo), sus preferencias, necesidades y sobre todo lo que está en tendencia. Tener en cuenta las actividades que están realizando la competencia, fortalezas, debilidades y estrategias de posicionamiento que aplica para de ese modo determinar por medio de estudios científicos la realidad externa en la que se encuentra la empresa, tema que servirá para proyectar el direccionamiento que tendrá la marca en el futuro. De igual forma, es necesario elaborar un autoanálisis de la empresa para conocer sus fortalezas, debilidades, estrategias aplicadas y reconocimiento de filosofía corporativa. (ILGO, 2019)

Paso 2: Construcción del diseño, este punto según el autor debe ser meticuloso porque de ello depende la psicología, misión y visión que la marca implemente; ya que con ella se busca que el consumidor se identifique y a la vez se diferencie del resto. Para complementar la construcción del diseño es necesario elaborar una lluvia de ideas o también llamado brainstorming para identificar los atributos únicos por citar algunos ejemplos elegancia, suntuosidad, innovación, alegría, etc., cualidades que la marca debe identificar durante su existencia. Para esto el autor aconseja que encontrar 4 05 atributos son los más recomendable para elaborar el concepto final y así obtener formar la personalidad de la marca. (ILGO, 2019)

Paso 3: Gozar de un puesto en la mente de los consumidores al conectar con sus necesidades respondiendo a estos criterios ¿Qué es la marca? ¿Qué ofrece? ¿Debido a que? y ¿Para qué?. (ILGO, 2019)

Paso 4: Producción de una identidad visual constituida en base a su logotipo, color, lema empresarial, olores y sonidos que se proyectara a largo plazo. Se debe crear un nombre, breve, único, especial, apropiado utilizando la estrategia del naming, para que formen un conglomerado de elementos que se mostraran en un solo conjunto como una identidad de marca. (ILGO, 2019)

Paso 5: Comunicación, un eje primordial en el cual se elabora una planeación estratégica para dar a conocer la marca, otorgándole protagonismo, resaltando sus atributos trayéndole beneficios como las interacciones, comentarios y referencias por parte de un público objetivo definido con anticipación. (ILGO, 2019)

\subsection{Renovación gráfica de una marca}

(Hoyos Ballesteros, 2016), los mercados van evolucionando y las empresas se ven en la necesidad de innovar y los consumidores quieren ver cosas novedosas y nuevas. Por esas exigencias la marca tiene que ir renovándose para que mantenga cierta sobriedad y transparencia a lo largo del tiempo.

Pero también existen procedencias para que una marca considere mejorar su imagen corporativa, entre ellas tenemos, los problemas a nivel de estructura perceptiva por parte de los consumidores, es decir que en la organización han existido problemas de posicionamiento o asociaciones que no corresponden a la proyección de la esencia de la empresa, producto o servicio. (Hoyos Ballesteros, 2016)

\section{Método}

\subsection{Tipo de investigación}

El tipo de investigación efectuada es de carácter cuantitativo, que va dirigida a los usuarios y ciudadanos con relación directa e indirecta al Consultorio Fisioterapéutico KAMB; para conocer la situación de la identidad e imagen corporativa de esta manera poder proponer una renovación que innove hacia algo más novedoso y a la vanguardia de los cambios globales.

\subsection{Participantes}

Para la ejecución de esta investigación el extracto del total de la población se ha tomado el $30 \%$ del total, que equivale a 120 personas que corresponderían a ese público externo al consultorio. A ello se aumentan la base de 10 usuarios del consultorio para conocer desde otra perspectiva de percepción de marca. Ambas cifras conforman un total de 130 encuestados.

\subsection{Instrumento}

Las técnicas de investigación utilizada en este proceso es la encuesta, que permite recoger datos sobre la gran mayoría de cuestionamientos sociales. Las preguntas planteadas son 17 preguntas que se encuentran divididas en dos partes, 8 preguntas dirigidas a los usuarios y 9 a los habitantes cercanos al consultorio. 


\subsection{Procedimiento de análisis estadístico}

En la primera parte del estudio descriptivo se realizó una encuesta de manera voluntaria a 10 pacientes del Consultorio Fisioterapéutico KAMB y a 120 personas aledañas al consultorio, para conocer la dos percepciones que tienen sobre la marca.

En primera encuesta contempla 10 preguntas, tres preguntas cerradas y siete preguntas con opción a aportar con otro criterio, la segunda contempla cinco preguntas cerradas y seis con opción a aportar criterios personales.

\section{Resultados}

\section{1 descripción de la muestra}

Dentro de la encuesta establecida a los usuarios del Consultorio Fisioterapéutico KAMB, la primera pregunta es: ¿Qué tipo de tratamiento se realiza usted en Consultorio Fisioterapéutico KAMB?, donde el tratamiento más escogido con el $30 \%$ fue lesión deportiva, cabe mencionar que existe un empate entre el $20 \%$ que se va a realizar masajes, el $20 \%$ se atiende las lesiones neurológicas, el $20 \%$ acude a sanar los traumas de la columna y el $10 \%$ que se remedia temas de post quirúrgicos, lo que implica que los usuarios de mayor impacto son jóvenes.

La segunda pregunta es: ¿Conoce usted que es una marca?, el $100 \%$ de las personas encuestadas señalan que, si conocen el concepto de una marca, lo que afirma que los usuarios conocen terminologías de mercadotecnia, esto puede ser porque todo el tiempo están rodeados por ellas.

La tercera es: ¿Con que relaciona usted a la marca?, Para los pacientes encuestados el $50 \%$ es logotipo lo más relacionado a la marca, seguido del $40 \%$ que considera a identidad como cercano, el $10 \%$ menciona que es empresa, finalmente $0 \%$ en comunicación y $0 \%$ en otros. Lo que implica que el logotipo es el término que más se relaciona a una marca, aquel que funciona como un signo identificador de la empresa. Es por ello que los usuarios lo reconocen inmediatamente al hablar de marcas.

La cuarta hace mención: ¿Consideraría necesario que KAMB Consultorio Fisioterapéutico realice un proceso de renovación de marca?, donde el $50 \%$ de los usuarios encuetados cree que si sería necesaria renovar la marca KAMB y el otro $50 \%$ menciona que no se necesita realizar dicho proceso. Lo que afirma que la opinión de los encuestados se encuentra divididas en partes iguales, marcando una tendencia hacia la posibilidad de que algo no está bien estructurado y se debe mejorar.

La quinta pregunta es: ¿Qué le cambiaria al logotipo de KAMB?. El $40 \%$ de los encuestados mencionan que es la tipografía es lo que se debe cambiar, en cambio el $10 \%$ señala que se debe mejorar el color, el $5 \%$ indica que no se hay nada que hacer en el símbolo y otros con el $0 \%$. Los pacientes afirman que la tipografía y el color son los elementos más importantes que se deben renovar en el logotipo del Consultorio Fisioterapéutico KAMB.

La sexta expresa: ¿Le gustaría recibir información sobre Consultorio Fisioterapéutico KAMB?, El $100 \%$ de los encuestados mencionan querer recibir una información de KAMB y el $0 \%$ no desean recibir noticias, Lo cual explica que existe buena acogida del servicio profesional de fisioterapia brindado en la entidad y, por ende, desean informarse de las actividades realizadas por el Consultorio Fisioterapéutico KAMB.

La séptima pregunta es: ¿Bajo qué medio le gustaría recibir la información?. El $40 \%$ escogió a Facebook como el medio para estar conectados con el consultorio KAMB, el $30 \%$ se decidió que sea Instagram, el $10 \%$ considera al correo electrónico como favorito, el $10 \%$ escoge a WhatsApp, $10 \%$ considera que vallas publicitarias es la indicada y por último están los ítems que no arrojaron resultados el $0 \%$ en Twitter, $0 \%$ de comunicación directa y $0 \%$ de tarjetas de presentación. Lo que afirma que los pacientes encuetados marcan una clara tendencia hacia las redes sociales, convirtiéndolo en una plataforma alternativa para mantenerse comunicado o interconectado.

En la segunda encuesta direccionada a los habitantes aledaños al Consultorio Fisioterapéutico KAMB, se entabla nueve preguntas, que unas mantienen relación con la anterior encuesta.

La primera pregunta manifiesta lo siguiente: ¿Conoce usted que es una marca?. El $90 \%$ de los encuestados conocen el concepto de marca frente a un $10 \%$ que no conoce el significado de una marca. La mayor cantidad conocen el concepto de marca, la relacionan a un producto o tienen una idea como tal. Lo que afirma la importancia que tiene el posicionamiento estratégico que ejecutan las grandes empresas hacia sus públicos objetivos, pues han logrado penetrar en la mente de los consumidores.

La segunda expresa: ¿Con que relaciona usted a la marca?. Al relacionar las marcas con una variedad de términos se obtuvo los siguientes datos el 53,33\% piensa que logotipo es la conexión más cercana que podría establecerse, el 33,33\% estipula que una empresa seria la indicada, un tercer lugar que lo ocupa el $13,33 \%$ cree que es la identidad, mientras que comunicación y otros reciben el $0,00 \%$. afirmando así que mayor relación que puede existir con la marca es el logotipo y la empresa; estos dos últimos términos trabajan conjuntamente para consolidar la identidad corporativa de una empresa.

La tercera pregunta es: ¿Conoce usted la marca KAMB Consultorio Fisioterapéutico?, El 82,5\% no conoce la marca Consultorio Fisioterapéutico KAMB frente al $17,5 \%$ comenta conocer dicha identidad. Una rotunda mayoría de ciudadanos expresan desconocer al Consultorio Fisioterapéutico KAMB, lo cual afirma que la marca no es conocida por las personas cercanas a la entidad. Entonces, el renovar la marca sería una alternativa considerable para que por medio del Branding lanzar, potencializar, reforzar la imagen gráfica de la identidad.

La cuarta pregunta: ¿Le gusta el rotulo de la marca KAMB?. Esta pregunta fue realizada a las 21 personas que indicaron conocer la marca KAMB donde expresaron lo siguiente, el 52,38\% afirma gustarle un poco el letrero 
que consta como referente de identidad del consultorio, el $28,57 \%$ comenta que no le gusta dicho rótulo y el 19,05\% conciben que si les agrada. Es relevante conocer que la gran mayoría de encuestados perciben un bajo nivel de agrado cuando visualizan el letrero que tiene Consultorio Fisioterapéuticos KAMB, del mismo modo, para algunos definitivamente no existe la más mínima afición. Un manejo de branding se hace inminente porque se debe sustentar el manejo de marca, también de la publicidad para generar mayor impacto que llame la atención de aquellos que les gusta un poco y los que aún no se sientes atraídos.

La quinta pregunta es: ¿Cree usted que el logotipo actual de KAMB representa bien al Consultorio Fisioterapéutico?. Los resultados fueron tomado solo a los 21 ciudadanos que conocen la marca KAMB, donde el 47,62\% menciona que quizás el logotipo represente bien al consultorio, un 28,57\% afirma que el símbolo no manifiesta adecuadamente a la entidad y el $23,81 \%$ plantea que el distintivo si representa de forma positiva a la entidad. Lo que implicaría que la renovación puede dar paso a un cambio en cuanto al posicionamiento, puesto que la gente no percibe una buena conexión entre el logo y la entidad, por ello es necesario reafirmar la marca con el propósito que no existan fallos en las percepciones de los habitantes.

La sexta pregunta plantea lo siguiente: ¿Consideraría necesario que KAMB Consultorio Fisioterapéutico realice un proceso de renovación de su marca?. Esta información fue recabada de las 21 personas que dicen conocer a KAMB donde el $100 \%$ de los encuestados muestran que si es necesario que KAMB se someta a una intervención de renovación de marca. Mientras que el 0,00\% dicen no estar de acuerdo con el cambio. Este cuestionamiento es muy relevante porque los ciudadanos en su totalidad, expresaron que, si es necesario realizar un proceso de renovación de marca en el Consultorio Fisioterapéutico KAMB, donde muchos indicaron razones específicas como: mejorar el diseño tanto en logotipo y en publicidad para que estos elementos tengan más visibilidad y llame la atención de los ciudadanos.

La séptima pregunta es: ¿Qué le cambiaria al logotipo KAMB?. El 57,14\% muestra que se debe generar un cambio total de elementos en el logotipo KAMB pero un $19,05 \%$ plantea que solo se debe modificar el color, el $14,29 \%$ cree que la mejora está en la tipografía, sin embargo, el $9,52 \%$ considera que el diseño es lo que se debe modificar y un $0,00 \%$ comenta que no debería existir ninguna trasformación. Donde la mayoría del público encuestado muestra que se debe realizar una renovación total de logotipo, tomando en cuenta primero en el color y luego su tipografía.

La octava pregunta es: ¿Le gustaría recibir información sobre consultorio Fisioterapéutico KAMB?. Para esta pregunta se toma la totalidad de la población cuyo número es 120 donde se muestra que el $81,67 \%$ desearía recibir información sobre el consultorio frente a un 18,33\% no quiere recibir ningún tipo de noticias al respecto de KAMB.
Lo que asevera que una gran mayoría de encuestados le gustaría recibir algún tipo de información, promociones o actividades que realiza la marca KAMB. Para aquello sería transcendental generar un mecanismo de comunicación para los posibles nuevos usuarios.

\section{Conclusiones}

Los habitantes que residen en los alrededores del consultorio no reconoce la marca KAMB ni la identifican como parte del consultorio. La marca tiene un posicionamiento muy frágil porque solo quienes estrictamente han tenido alguna relación directa con la entidad la recuerdan y puede emitir una opinión valiosa que consolida la propuesta planteada en esta investigación, puesto que denotan que renovar es una oportunidad para la empresa porque quieren ver una marca que, se muestre con personalidad, fresca, fuerte y que transmita y la que actualmente tiene no proyecta esas cualidades, es más la publicidad externa no está bien direccionada según los encuestados.

Para identificar la cantidad de personas que reconocieron la marca. Naturalmente constan los 8 pacientes que la entidad maneja diariamente, también se tomó en cuenta al público externo y de ellos exactamente 21 personas de un total de 120 dijeron identificar la marca Consultorio Fisioterapéutico KAMB. Sin embargo, eso no impidió que la mayoría expresara su inconformidad hacia el logotipo y la publicidad externa de KAMB, este que está compuesto de las iniciales del nombre de su propietaria que a la vez es fisioterapista.

El público objetivo o target reconocido en esta investigación es un público diverso que oscila entre 13 a 30 años de edad, que más se someten a tratamientos de fisioterapia en el consultorio y la arista más contundente es por cuestiones deportivas y en el publico externo predomino en sus alrededores la gente joven.

Las estrategias se han planteado tomando como eje principal al branding, donde la marca no es un logotipo, sino que ella usa a este identificador como un instrumento, para que por medio de él transmitir valores, filosofía empresarial, personalidad que una sola palabra se resume como identidad corporativa. Con el branding el diseño de una renovada marca conlleva una serie de percepciones intangibles como el posicionamiento y otras tangibles como su nombre y su línea grafica que al visualizar, escuchar y palpar tenga una armonía sutil. La propuesta se realizó para que la nueva marca presente una identidad, ser aplicada con especificaciones legales y legitimad donde el impulso es generado por la publicidad. También se potencia las plataformas virtuales como Facebook, Instagram, Vallas Publicitarias, Correo Electrónico para tener presencia en el mundo virtual.

\section{Recomendaciones}

Forjar por medio del diseño, comunicación y colocación, una publicidad responsable para obtener una identidad más sólida con el paso del tiempo, en base a la exposición 
de la marca renovada que llegue con su esencia a muchas más personas con la idea de que se ellos se conviertan en futuros clientes del consultorio.

Mejorar la estructura de las instalaciones actuales de KAMB, para ofrecer un servicio con más comodidad.

Estar siempre a la vanguardia en cuanto a los aparatos tecnológicos.

Emitir tarjetas de presentación como método de cercanía con el cliente.

Colocar un letrero luminoso que llame la atención de los ciudadanos en la Av.47 de Manta. Actualmente existe uno allí, pero no es visible para los transeúntes y conductores.

Crear una página de Facebook e Instagram para interactuar con más personas pero que exista un manejo de contenido profesional.

\section{Referencias Bibliográficas}

Alejandro Razak. (2019). Qué es y para qué sirve el branding [+PDF] | Branfluence.

Alicia Anabel Aucapiña Alvarado. (2017). "Branding: propuesta de estilización de marca y material promocional para el área nacional de recreación Isla Santay ubicada en la provincia del Guayas". , 88.

Caceres Gutierrez Amparo. (2012). Branding Emocional । Cáceres Gutierrez I TECCIENCIA.

Capriotti, P. (2010). Branding corporativo. Gestión estratégica de la identidad corporativa. Comunicación, $O(27), 15-22$.

Cubur Luis, C., y Fernando, L. (2015). Diseño de una guía de aplicación de imagotipo para estandarizar la imagen gráfica en materiales y documentos digitales e impresos del departamento de investigación y desarrollo del Galileo Education System -GES- (Tesis Doctoral no publicada).

Delgado Gentili Luis Armando. (2012). Evolución del concepto de construcción de marca a través de una estrategia de branding., 157.

Del-Rio, J., Cardona-Arbelaez, D., y Simancas-Trujillo, R. (2017, nov). Propuesta de un modelo teórico de branding para el posicionamiento de la marca universitaria. Revista ESPACIOS, 38(53).

Duarte, E. V., y Contreras, J. A. O. (2014, jul). Estética e identidad corporativa. Aibi revista de investigación, administración e ingeniería, 50-55.

Evangelista, L. T., y Baca, Y. M. A. (2018). Uso de la teoría del color para mejorar la atención visual en estudiantes del segundo grado de secundaria de la I.E. San Martín de Socabaya, Arequipa 2017. , 98.

Flores Calderon Blanca, B. (2015). Guía para diseñar una marca. Barcelona: Editorial UOC.

Grau, X. (2011). Naming. ¿Cómo crear un buen nombre? Editorial UOC.

Hernandez Daniel. (2017). Renovación de la marca siscomservice (Tesis Doctoral no publicada).

Hernandez White Raquel. (2012). Branding sustentable.
Hoyos Ballesteros, R. (2016). Branding: el arte de marcar corazones. Bogotá: Ecoe Ediciones.

ILGO. (2019). Como crear una marca.

Lueza, J. M. (2018). Comunicación estratégica: diseño de la identidad corporativa. Volumen 1: identidad vs. imagen. Editorial Digital del Tecnológico de Monterrey.

Lupton, E., y Phillips, J. C. (2016). Diseño gráfico: nuevos fundamentos. Barcelona: Editorial Gustavo Gili.

Medina-Aguerrebere, P. (2014). Marca y comunicación empresarial. Editorial UOC.

Meldini Adriana. (2015). Branding emocional para deseos personales (Tesis Doctoral no publicada).

Olins, W. (2015). Brand New: la esencia de las futuras marcas. Lima: Universidad Peruana de Ciencias Aplicadas (UPC).

Olvera, E. G. C., y Zambrano, Z. Y. J. (2019). Identidad visual corporativa en el posicionamiento de la unidad de producción alimenticia de la economía popular solidaria ubicada en Guayaquil ASOPLATCA, 2018. , 190.

Ortegón Costázar, L. (2014). Gestión de marca: conceptualización, diseño, registro, construcción y evaluación. Bogotá: Editorial Politécnico Grancolombiano.

Perez Ortega Andres. (2008). Marca Personal: Cómo convertirse en la opción preferente. ESIC Editorial.

Santamaria Luiggi. (2013, dec). Branding: Definición e Historia.

Soza Angie. (2019). Tipos de Branding - Agencia de Publicidad y Marketing.

Valenzuela Tamayo Alejandro. (2013). Branding construccion de marcas. , 39.

Valiente Alber, S. (2016). Marcas sonrientes: humor $y$ engagement en publicidad. Barcelona: Editorial UOC.

Velilla, J. (2010). Branding: tendencias y retos en la comunicación de marca. Barcelona: Editorial UOC.

Zapana, D. F. C., y Salvador, W. K. I. (2018). Presentado por las bachilleres. , 113 .

Recibido: 13 de agosto de 2019

Aceptado: 3 de junio de 2020 